\title{
Fundamental groups of algebraic fiber spaces
}

\author{
Ichiro Shimada
}

\begin{abstract}
Let $f: E \rightarrow B$ be a dominant morphism, where $E$ and $B$ are smooth irreducible complex quasi-projective varieties. Suppose that the general fiber $F_{b}$ of $f$ is connected. We present an algebro-geometric condition under which the boundary homomorphism $\partial: \pi_{2}(B) \rightarrow$ $\pi_{1}\left(F_{b}\right)$ is well-defined, and makes the sequence

$$
\pi_{2}(B) \rightarrow \pi_{1}\left(F_{b}\right) \rightarrow \pi_{1}(E) \rightarrow \pi_{1}(B) \rightarrow 1
$$
\end{abstract}

exact. As an application, we calculate the fundamental group of the complement to the dual hypersurface of a smooth projective curve.

Mathematics Subject Classification (2000). 14F35, 14 D05.

Keywords. Fundamental group, second homotopy group, algebraic fiber space, homotopy exact sequence, dual hypersurface.

\section{Introduction}

We work over the complex number field $\mathbb{C}$.

Let $\bar{E}$ and $B$ be smooth irreducible quasi-projective varieties, and let

$$
\bar{f}: \bar{E} \rightarrow B
$$

be a projective surjective morphism. Throughout this paper, we assume that the general fiber of $\bar{f}$ is connected. Let $Z$ be a reduced hypersurface of $\bar{E}$. We denote by $E$ the complement $\bar{E} \backslash Z$ to $Z$ in $\bar{E}$, and by $f$ the restriction $\bar{f} \mid E$ of $\bar{f}$ to $E$. Then

$$
f: E \rightarrow B
$$

is a dominant morphism with the general fiber being a smooth irreducible quasiprojective variety. For a point $a \in B$, we denote by $\bar{F}_{a}$ the fiber $\bar{f}^{-1}(a)$, and by $Z_{a}$ the scheme-theoretic intersection of $\bar{F}_{a}$ and $Z$. We put

$$
F_{a}:=f^{-1}(a)=\bar{F}_{a} \backslash Z_{a} .
$$

We choose a general point $b$ of $B$, and a point $\tilde{b}$ of $F_{b}$. Let

$$
i: F_{b} \hookrightarrow E
$$


denote the inclusion morphism. We will study the homomorphisms

$$
i_{*}: \pi_{1}\left(F_{b}, \tilde{b}\right) \rightarrow \pi_{1}(E, \tilde{b}) \quad \text { and } \quad f_{*}: \pi_{1}(E, \tilde{b}) \rightarrow \pi_{1}(B, b)
$$

on the topological fundamental groups induced by the morphisms $i$ and $f$. By investigating the homotopy lifting property of the morphism $f$, we will show the following:

Theorem 1.1. Suppose that $\bar{f}: \bar{E} \rightarrow B$ and $Z$ satisfy the following conditions.

(C1) Every irreducible component of the hypersurface $Z$ is mapped surjectively onto $B$ by $\bar{f}$.

(C2) The locus Sing $\bar{f} \subset \bar{E}$ of the critical points of $\bar{f}$ is of codimension $\geq 3$ in $\bar{E}$.

(C3) The locus $\left\{a \in B \mid Z_{a}\right.$ is not reduced $\}$ is contained in a Zariski closed subset of codimension $\geq 2$ in $B$.

Then the boundary homomorphism

$$
\partial: \pi_{2}(B, b) \rightarrow \pi_{1}\left(F_{b}, \tilde{b}\right)
$$

is well-defined, and the sequence

$$
\pi_{2}(B, b) \stackrel{\partial}{\longrightarrow} \pi_{1}\left(F_{b}, \tilde{b}\right) \stackrel{i_{*}}{\longrightarrow} \pi_{1}(E, \tilde{b}) \stackrel{f_{*}}{\longrightarrow} \pi_{1}(B, b) \longrightarrow 1
$$

is exact.

In fact, Nori [8, Lemma $1.5(\mathrm{C})]$ has proved that, under a condition milder than (C1)-(C3), the sequence

$$
\pi_{1}\left(F_{b}, \tilde{b}\right) \stackrel{i_{*}}{\longrightarrow} \pi_{1}(E, \tilde{b}) \stackrel{f_{*}}{\longrightarrow} \pi_{1}(B, b) \longrightarrow 1
$$

is exact.

A special case of Theorem 1.1, where $B$ is an affine space $\mathbb{A}^{N}, \bar{E}$ is a product $\bar{F} \times$ $\mathbb{A}^{N}$ of a smooth irreducible projective variety $\bar{F}$ and $\mathbb{A}^{N}$, and $\bar{f}$ is the projection, was proved in [10].

As an application, we will calculate the fundamental group of the complement to the dual hypersurface of a smooth projective curve.

Let $C$ be a compact Riemann surface of genus $g>0$, and $L \rightarrow C$ a line bundle of degree $d>2 g+1$. Then the complete linear system $|L|$ embeds $C$ into the projective space

$$
P_{L}:=\mathbb{P}^{*} H^{0}(C, L)
$$

parameterizing all hyperplanes of the vector space $H^{0}(C, L)$ of dimension $d-$ $g+1$. Let $C_{L}$ denote the image of this embedding. We denote by $C_{L}^{\vee}$ the dual hypersurface of $C_{L}$ in the dual projective space $P_{L}^{\vee}$ of $P_{L}$. Let $B(C, d)$ be the braid group on $d$ strings on the Riemann surface $C$; that is, $B(C, d)$ is the fundamental group of the space

$$
\operatorname{Sym}^{d} C \backslash \Delta_{C}^{d},
$$

where $\operatorname{Sym}^{d} C$ is the symmetric product of $d$ copies of $C$, which parameterizes all effective divisors of degree $d$ on $C$, and $\Delta_{C}^{d}$ is the hypersurface of $\operatorname{Sym}^{d} C$ parameterizing all non-reduced effective divisors of degree $d$. 
Theorem 1.2. If $L$ corresponds to a general point of the Picard variety $\operatorname{Pic}^{d}(C)$ of isomorphism classes of line bundles of degree $d$ on $C$, then $\pi_{1}\left(P_{L}^{\vee} \backslash C_{L}^{\vee}\right)$ is isomorphic to the kernel of the natural homomorphism

$$
B(C, d) \longrightarrow H_{1}(C, \mathbb{Z}) .
$$

Theorem 1.2 was stated in [3]. However the proof in [3] seems to be incomplete, because the family of the complements $P_{L}^{\vee} \backslash C_{L}^{\vee}$ is, in general, not locally trivial over $\operatorname{Pic}^{d}(C)$. Another proof of Theorem 1.2 was also given in [7], but the proof is sketchy.

A finite presentation of $B(C, d)$ is given in [1], [2] and [9]. A finite presentation of the kernel of the natural homomorphism from $B(C, d)$ to $H_{1}(C, \mathbb{Z})$ is given in $[6]$ and $[7]$.

This paper is organized as follows. In $\S 2$, we collect miscellaneous definitions and lemmas that will be used in this paper. In $\S 3$, we state Nori's lemma $[8$, Lemma $1.5(\mathrm{C})]$, and give a proof which is different from Nori's original proof, and uses a similar idea as the proof of Theorem 1.1. In $\S 4$, we define the boundary homomorphism $\partial$ from $\pi_{2}(B)$ to $\pi_{1}\left(F_{b}\right)$, and in $\S 5$, we prove Theorem 1.1. In $\S 6$, we prove Theorem 1.2.

The author would like to thank Professor Vik. S. Kulikov for helpful discussions.

\section{Notation and terminologies}

(1) We consider algebraic varieties with the complex topology unless otherwise stated. An algebraic morphism $\phi: X \rightarrow Y$ is said to be locally trivial over $Y$ if it is locally trivial over $Y$ as a continuous map in the complex topology.

(2) The Zariski closure of a subset $A$ of an algebraic variety is denoted by $A^{=}$.

(3) For an algebraic variety $X$, we denote by $\operatorname{Sing} X$ the singular locus of $X$. For a morphism $\phi: X \rightarrow Y$ with $X$ and $Y$ smooth, we denote by Sing $\phi \subset X$ the locus of critical points of $\phi$.

(4) We denote by $I$ the closed interval $[0,1]$ in $\mathbb{R}$. For a subset $A$ of $I^{k}$, we denote by $A^{\circ}$ the interior of $A$ in $\mathbb{R}^{k}$, by $(A)^{-}$or $\bar{A}$ the closure of $A$, and by $\partial A$ the boundary $\bar{A} \backslash A^{\circ}$ of $A$.

(5) Let $p$ be a point of a topological space $X$, and $u:\left(I^{k}, \partial I^{k}\right) \rightarrow(X, p)$ a continuous map. We denote by $[u]$ the element of $\pi_{k}(X, p)$ represented by $u$. The constant map from $I^{k}$ to the point $p$ is denoted by $0_{p}$.

(6) Let $\phi: X \rightarrow Y$ and $\psi: Z \rightarrow Y$ be continuous maps. We say that $\phi$ is locally trivial over $\psi$ or over $Z$ if the pull-back

$$
\psi^{*} \phi: \psi^{*} X=X \times_{Y} Z \rightarrow Z
$$

of $\phi$ by $\psi$ is locally trivial over $Z$. 


\section{Preliminaries}

\subsection{Transversality}

Let $M$ and $N$ be connected $\mathcal{C}^{\infty}$ differentiable manifolds, and $S$ a closed submanifold of $N$, which may have several connected components of various dimensions. Let $Y$ be a smooth irreducible quasi-projective variety, and $T$ a closed, reduced (and possibly reducible) subvariety of $Y$.

Definition 2.1. (1) We say that a $\mathcal{C}^{\infty}$-map $\phi: M \rightarrow N$ intersects $S$ transversely if, for any $p \in \phi^{-1}(S)$, we have

$$
(d \phi)_{p}\left(T_{p} M\right)+T_{\phi(p)} S=T_{\phi(p)} N .
$$

(2) We say that a continuous map $v: I^{k} \rightarrow N$ intersects $S$ transversely if there exists an open subset $U$ of $I^{k}$ satisfying

$$
v^{-1}(S) \subset U \subset \bar{U} \subset\left(I^{k}\right)^{\circ}
$$

such that the restriction

$$
v \mid U: U \rightarrow N
$$

of $v$ to $U$ is a $\mathcal{C}^{\infty}$-map that intersects $S$ transversely in the sense of (1) above.

(3) We say that a $\mathcal{C}^{\infty}$-map $\varphi: M \rightarrow Y$ intersects $T$ transversely if the following hold;

- $\varphi^{-1}(\operatorname{Sing} T)=\emptyset$, so that $\varphi$ can be regarded as a $\mathcal{C}^{\infty}$-map to $Y \backslash \operatorname{Sing} T$, which contains $T \backslash \operatorname{Sing} T$ as a closed submanifold, and

- as a $\mathcal{C}^{\infty}$-map to $Y \backslash \operatorname{Sing} T, \varphi$ intersects the closed submanifold $T \backslash \operatorname{Sing} T$ transversely in the sense of (1) above.

(4) We say that a continuous map $w: I^{k} \rightarrow Y$ intersects $T$ transversely if there exists an open subset $U$ of $I^{k}$ satisfying

$$
w^{-1}(T) \subset U \subset \bar{U} \subset\left(I^{k}\right)^{\circ}
$$

such that the restriction $w \mid U$ of $w$ to $U$ is a $\mathcal{C}^{\infty}$-map that intersects $T$ transversely in the sense of (3) above.

Let $A, V$ and $W$ be open subsets of $M$ such that the closure $\bar{V}$ of $V$ in $M$ is compact and contained in $W$. Suppose that a distance function

$$
d: N \times N \rightarrow \mathbb{R}_{\geq 0}
$$

on $N$ is given. Using the approximation theorem of continuous maps by $\mathcal{C}^{\infty}$-maps and the elementary transversality theorem ([5]), we can easily prove the following:

Lemma 2.2. Let $\phi_{0}: M \rightarrow N$ be a continuous map. Suppose that the restriction $\phi_{0} \mid A$ of $\phi_{0}$ to $A$ is a $\mathcal{C}^{\infty}$-map intersecting $S$ transversely. Then, for any positive real number $\varepsilon$, there exists a continuous map $\phi_{1}: M \rightarrow N$ with the following properties; 
- there exists a homotopy from $\phi_{0}$ to $\phi_{1}$ that is stationary on $M \backslash W$,

- the restriction $\phi_{1} \mid(A \cup V)$ of $\phi_{1}$ to $A \cup V$ is a $\mathcal{C}^{\infty}$-map intersecting $S$ transversely, and

- $d\left(\phi_{0}(x), \phi_{1}(x)\right) \leq \varepsilon$ for any $x \in M$.

Starting from $T_{0}:=T$, we put

$$
T_{i+1}:=\operatorname{Sing} T_{i},
$$

where $T_{i}$ is considered as a subscheme of $Y$ with the reduced structure. Then $T_{n}$ is empty for $n$ large enough. By definition, $T_{i} \backslash T_{i+1}$ is a closed submanifold of $Y \backslash T_{i+1}$. Applying Lemma 2.2 to these closed submanifolds repeatedly, we obtain the following:

Corollary 2.3. Let $\varphi_{0}: M \rightarrow Y$ be a continuous map such that $\varphi_{0} \mid A$ is a $\mathcal{C}^{\infty}$ map intersecting $T$ transversely. If

$$
\operatorname{dim}_{\mathbb{R}} M+2 \operatorname{dim} \operatorname{Sing} T<2 \operatorname{dim} Y,
$$

then there exists a continuous map $\varphi_{1}: M \rightarrow Y$ with the following properties;

- there exists a homotopy from $\varphi_{0}$ to $\varphi_{1}$ that is stationary on $M \backslash W$, and

- the restriction $\varphi_{1} \mid(A \cup V)$ of $\varphi_{1}$ to $A \cup V$ is a $\mathcal{C}^{\infty}$-map intersecting $T$ transversely.

Corollary 2.4. Let $w_{0}: I^{k} \rightarrow Y$ be a continuous map such that $w_{0}^{-1}(T)$ is contained in $\left(I^{k}\right)^{\circ}$. If

$$
k+2 \operatorname{dim} \operatorname{Sing} T<2 \operatorname{dim} Y,
$$

then there is a continuous map $w_{1}: I^{k} \rightarrow Y$ intersecting $T$ transversely that is homotopic to $w_{0}$ relative to $\partial I^{k}$.

\subsection{Local triviality of a $\mathcal{C}^{\infty}$-map}

Let $M$ and $N$ be $\mathcal{C}^{\infty}$ manifolds, and $R$ a closed submanifold of $M$, which may have several connected components of various dimensions. Let $\phi: M \rightarrow N$ be a $\mathcal{C}^{\infty}$-map.

Lemma 2.5. Let $K$ be a non-empty compact subset of $M$ contained in the inverse image $\phi^{-1}(p)$ of a point $p \in N$. Suppose that $\phi$ is smooth at every point of $K$, and that the restriction $\phi \mid R$ of $\phi$ to $R$ is smooth at every point of $R \cap K$. Then there exist open neighborhoods $V$ of $p$ in $N$ and $U$ of $K$ in $M$ contained in $\phi^{-1}(V)$ such that the pair of the $\mathcal{C}^{\infty}$-maps

$$
(\phi|U, \phi| U \cap R):(U, U \cap R) \rightarrow V
$$

is locally trivial. 


\subsection{The topological discriminant locus of an algebraic morphism}

Let $X$ and $Y$ be smooth irreducible quasi-projective varieties, and $\phi: X \rightarrow Y$ a dominant morphism.

Definition 2.6. The topological discriminant locus $\Sigma_{\phi}$ is the minimal Zariski closed subset among the Zariski closed subsets $\Sigma$ of $Y$ with the following properties;

- $\Sigma$ contains the locus $\phi(\operatorname{Sing} \phi)$ of the critical values of $\phi$, and

- $\phi$ is locally trivial over $Y \backslash \Sigma$. (See Notation and terminologies (1) and (6).)

There always exists the topological discriminant locus $\Sigma_{\phi}$ (possibly $\Sigma_{\phi}=\emptyset$ ) such that $\Sigma_{\phi} \neq Y([8$, Lemma $1.5(\mathrm{~A})])$.

\subsection{Bertini's Theorem}

See [4, Theorem 1.1] for the proof of the following:

Lemma 2.7. Let $X \subset \mathbb{P}^{N}$ be an irreducible quasi-projective variety of codimension $c$ in $\mathbb{P}^{N}$, and let $\Lambda$ be a general linear subspace of $\mathbb{P}^{N}$ with dimension $c+1$.

(1) The scheme-theoretic intersection $\Lambda \cap X$ is an irreducible curve.

(2) Suppose that $\operatorname{dim} \operatorname{Sing} X \leq \operatorname{dim} X-2$. Then $\Lambda \cap X$ is smooth.

(3) Suppose that $X$ is smooth. Then the inclusion $\Lambda \cap X \hookrightarrow X$ induces a surjective homomorphism from $\pi_{1}(\Lambda \cap X)$ to $\pi_{1}(X)$.

\section{Nori's lemma}

Let $f: E \rightarrow B$ be as in $\S 1$. We denote by $\Sigma_{f} \subset B$ the topological discriminant locus of $f$. We will consider $\Sigma_{f}$ as a reduced subscheme of $B$. In particular, we have

$$
\operatorname{dim} \operatorname{Sing} \Sigma_{f} \leq \operatorname{dim} B-2 .
$$

Since we have assumed that the general fiber of $\bar{f}$ is connected, $F_{a}$ is connected for any $a \in B \backslash \Sigma_{f}$. Since $b \in B$ is general, we can assume $b \notin \Sigma_{f}$.

Proposition 3.1 ([8], Lemma 1.5 (C)). Suppose that the Zariski closed subset

$$
\Xi_{1}:=\left\{a \in B \mid F_{a} \backslash\left(F_{a} \cap \operatorname{Sing} f\right)=\emptyset\right\}=
$$

is of codimension $\geq 2$ in $B$. Then the sequence

$$
\pi_{1}\left(F_{b}, \tilde{b}\right) \stackrel{i_{*}}{\longrightarrow} \pi_{1}(E, \tilde{b}) \stackrel{f_{*}}{\longrightarrow} \pi_{1}(B, b) \longrightarrow 1
$$

is exact. 
We hope that the proof of Proposition 3.1 given below will be helpful in understanding the idea of the proof of Theorem 1.1. First we prove the following lemmas, which will be also used in $\S 4$.

Lemma 3.2. Let $\xi: I \rightarrow B \backslash \Sigma_{f}$ be a path. Suppose that points $p_{0} \in F_{\xi(0)}$ and $p_{1} \in F_{\xi(1)}$ are given. Then there exists a lift $\widetilde{\xi}: I \rightarrow E \backslash f^{-1}\left(\Sigma_{f}\right)$ of $\xi$ that satisfies $\widetilde{\xi}(0)=p_{0}$ and $\widetilde{\xi}(1)=p_{1}$.

Proof. Since $f$ is locally trivial over $\xi$, the pull-back $\xi^{*} E \rightarrow I$ of $f$ by $\xi$ is trivial. Since the fiber of $\xi^{*} E \rightarrow I$ is connected, the existence of the lift $\widetilde{\xi}$ connecting $p_{0}$ and $p_{1}$ follows.

Lemma 3.3. Let $v: I \times I \rightarrow B$ be a continuous map that intersects $\Sigma_{f}$ transversely and satisfies $v^{-1}\left(\Xi_{1}\right)=\emptyset$. Suppose that we are given a lift

$$
\left(v_{0}\right)^{\sim}: I \times\{0\} \rightarrow E
$$

of the restriction $v_{0}:=v \mid I \times\{0\}$ of $v$ to $I \times\{0\}$. Then there exists a lift

$$
\widetilde{v}: I \times I \rightarrow E
$$

of $v$ such that the restriction $\widetilde{v} \mid I \times\{0\}$ of $\widetilde{v}$ to $I \times\{0\}$ is equal to $\left(v_{0}\right) \sim$, and that $\widetilde{v}^{-1}(\operatorname{Sing} f)$ is empty.

Proof. Since $v$ intersects $\Sigma_{f}$ transversely, $v^{-1}\left(\Sigma_{f}\right)$ is a finite set of points of $(I \times I)^{\circ}$. In particular, the image of $\left(v_{0}\right)^{\sim}$ is disjoint from Sing $f$, because Sing $f \subset f^{-1}\left(\Sigma_{f}\right)$ by the definition of the topological discriminant locus. We put

$$
v^{-1}\left(\Sigma_{f}\right):=\left\{p_{1}, \ldots, p_{N}\right\} \text {. }
$$

Since $v\left(p_{i}\right) \notin \Xi_{1}$, there exists at least one point of $F_{v\left(p_{i}\right)}$ at which $f$ is smooth. Hence there is an open neighborhood $U_{i}$ of $v\left(p_{i}\right)$ on which a holomorphic local section

$$
s_{i}: U_{i} \rightarrow f^{-1}\left(U_{i}\right)
$$

of $f$ is defined. Note that $s_{i}\left(U_{i}\right) \cap \operatorname{Sing} f=\emptyset$. There exists a homeomorphism

$$
\psi: I \times I \stackrel{\sim}{\rightarrow} I \times I
$$

such that $\psi \mid I \times\{0\}$ is the identity map of $I \times\{0\}$, and that

$$
\psi\left(p_{i}\right)=(i /(N+1), 1 / 2) \in(I \times I)^{\circ} \quad \text { for every } \quad p_{i} \in v^{-1}\left(\Sigma_{f}\right) .
$$

We choose a sufficiently small positive real number $\rho$, and put

$$
\begin{aligned}
\Delta_{i}^{\prime} & :=\left\{(x, y) \in I \times I \mid(x-i /(N+1))^{2}+(y-1 / 2)^{2} \leq \rho^{2}\right\}, \\
Z_{i}^{\prime} & :=\{(i /(N+1), t) \in I \times I \mid t \in[0,1 / 2-\rho]\} .
\end{aligned}
$$


We then put

$$
\Delta_{i}:=\psi^{-1}\left(\Delta_{i}^{\prime}\right), \quad Z_{i}:=\psi^{-1}\left(Z_{i}^{\prime}\right) \quad \text { and } \quad Q:=(I \times\{0\}) \cup \bigcup_{i=1}^{N}\left(\Delta_{i} \cup Z_{i}\right)
$$

Since $\rho$ is small enough, we can assume that $v\left(\Delta_{i}\right) \subset U_{i}$. Therefore a lift

$$
\left(v \mid \Delta_{i}\right)^{\sim}: \Delta_{i} \rightarrow E
$$

of $v \mid \Delta_{i}$ can be defined by

$$
\left(v \mid \Delta_{i}\right)^{\sim}:=s_{i} \circ\left(v \mid \Delta_{i}\right)
$$

Note that

$$
\left(v \mid \Delta_{i}\right)^{\sim}\left(\Delta_{i}\right) \cap \operatorname{Sing} f=\emptyset .
$$

We put

$$
(I \times\{0\}) \cap Z_{i}=\left\{r_{i}\right\}, \quad \Delta_{i} \cap Z_{i}=\left\{q_{i}\right\}, \quad \text { and } \quad \tilde{q}_{i}:=\left(v \mid \Delta_{i}\right)^{\sim}\left(q_{i}\right) \in E .
$$

Since $Z_{i} \cap v^{-1}\left(\Sigma_{f}\right)=\emptyset, v\left(Z_{i}\right)$ is contained in $B \backslash \Sigma_{f}$. By Lemma 3.2, we have a lift

$$
\left(v \mid Z_{i}\right)^{\sim}: Z_{i} \rightarrow E
$$

of $v \mid Z_{i}$ such that $\left(v \mid Z_{i}\right) \sim\left(r_{i}\right)=\left(v_{0}\right) \sim\left(r_{i}\right)$ and $\left(v \mid Z_{i}\right) \sim\left(q_{i}\right)=\tilde{q}_{i}$. Then a lift

$$
(v \mid Q)^{\sim}: Q \rightarrow E
$$

of $v \mid Q$ can be defined by

$$
(v \mid Q)^{\sim}(p):= \begin{cases}\left(v_{0}\right)^{\sim}(p) & \text { if } p \in I \times\{0\}, \\ \left(v \mid Z_{i}\right)^{\sim}(p) & \text { if } p \in Z_{i}, \\ \left(v \mid \Delta_{i}\right)^{\sim}(p) & \text { if } p \in \Delta_{i} .\end{cases}
$$

Note that $v^{-1}\left(\Sigma_{f}\right)$ is contained in the interior of $Q$. Hence $f$ is locally trivial over the restriction $v \mid\left((I \times I) \backslash Q^{\circ}\right)$ of $v$ to $(I \times I) \backslash Q^{\circ}$. Since $Q$ is a strong deformation retract of $I \times I$, we can extend $(v \mid Q)^{\sim}$ to a lift

$$
\widetilde{v}: I \times I \rightarrow E
$$

of $v$. Since Sing $f$ is contained in $f^{-1}\left(\Sigma_{f}\right)$, we have

$$
\widetilde{v}^{-1}(\operatorname{Sing} f) \subset v^{-1}\left(\Sigma_{f}\right) \subset \cup \Delta_{i} .
$$

Since $\widetilde{v} \mid \Delta_{i}$ coincides with $\left(v \mid \Delta_{i}\right)^{\sim}$, we have $\widetilde{v}^{-1}(\operatorname{Sing} f)=\emptyset$ by (3.1). By construction, $\widetilde{v} \mid I \times\{0\}$ coincides with $\left(v_{0}\right) \sim$.

Proof of Proposition 3.1. The surjectivity of $f_{*}$ follows immediately from the connectedness of the general fiber of $f$. It is also obvious that $\operatorname{Im} i_{*}$ is contained in $\operatorname{Ker} f_{*}$. Hence all we have to prove is $\operatorname{Ker} f_{*} \subseteq \operatorname{Im} i_{*}$. 


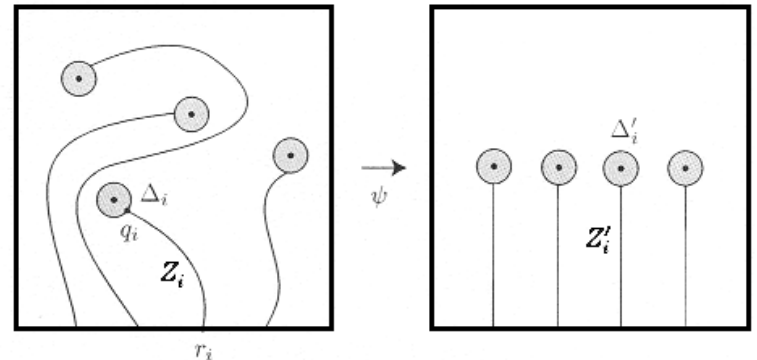

FIG. 3.1. The curve segment $Z_{i}$ connecting $r_{i}$ and $q_{i}$

Suppose that a loop

$$
u:(I, \partial I) \rightarrow(E, \tilde{b})
$$

represents an element $[u]$ of $\operatorname{Ker} f_{*}$. By Corollary 2.4, we can assume that $u^{-1}\left(f^{-1}\left(\Sigma_{f}\right)\right)=\emptyset$. There is a homotopy

$$
w:(I, \partial I) \times I \rightarrow(B, b)
$$

from the loop

$$
f \circ u:(I, \partial I) \rightarrow(B, b)
$$

to the constant loop $0_{b}$. We have $w^{-1}\left(\Sigma_{f}\right) \subset(I \times I)^{\circ}$. Note that $\Xi_{1} \subset \Sigma_{f}$. By Corollary 2.4 and the assumption on the codimension of $\Xi_{1}$, we can assume that $w$ intersects $\Sigma_{f}$ transversely, and that $w^{-1}\left(\Xi_{1}\right)$ is empty. We put

$$
K:=(\partial I \times I) \cup(I \times\{0\}) .
$$

We can define a lift

$$
(w \mid K)^{\sim}: K \rightarrow E
$$

of $w \mid K$ by

$$
(w \mid K)^{\sim}(p):= \begin{cases}\tilde{b} & \text { if } p \in \partial I \times I, \\ u(t) & \text { if } p=(t, 0) \in I \times\{0\} .\end{cases}
$$

There is a homeomorphism

$$
\zeta:(I \times I, I \times\{0\}) \stackrel{\sim}{\rightarrow}(I \times I, K)
$$

that is locally diffeomorphic at each point of $(w \circ \zeta)^{-1}\left(\Sigma_{f}\right)$. Then $w \circ \zeta$ intersects $\Sigma_{f}$ transversely. We define a lift of $(w \circ \zeta) \mid I \times\{0\}$ to be the composite $(w \mid K)^{\sim}$ 。 $(\zeta \mid I \times\{0\})$. This lift can be extended to a lift $(w \circ \zeta)^{\sim}$ of $w \circ \zeta$ by Lemma 3.3. Hence we can extend $(w \mid K)^{\sim}$ to a lift

$$
\widetilde{w}:(I, \partial I) \times I \rightarrow(E, \tilde{b})
$$

of $w$ by defining $\widetilde{w}:=(w \circ \zeta)^{\sim} \circ \zeta^{-1}$. We define a loop 

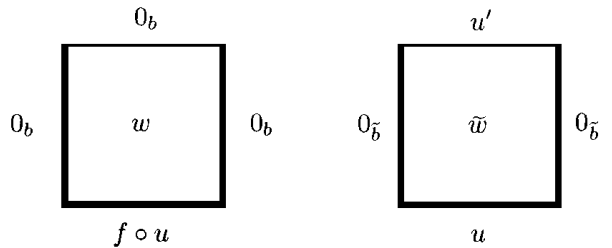

FIG. 3.2. The homotopy $w$ and its lift $\widetilde{w}$

$$
u^{\prime}:(I, \partial I) \rightarrow\left(F_{b}, \tilde{b}\right)
$$

to be the restriction $\widetilde{w} \mid I \times\{1\}$ of $\widetilde{w}$ to $I \times\{1\}$. Then $\widetilde{w}$ yields a homotopy from the given loop $u$ in $E$ to the loop $u^{\prime}$ in $F_{b}$. Therefore we have

$$
[u]=\left[u^{\prime}\right] \in \operatorname{Im} i_{*} .
$$

Thus Ker $f_{*} \subseteq \operatorname{Im} i_{*}$ is proved.

\section{The boundary homomorphism}

We consider the following condition on $f: E \rightarrow B$.

Condition (S). There exists a locally closed smooth irreducible subvariety $S$ of $E$ with the following properties.

Let $g: S \rightarrow B$ denote the restriction of $f$ to $S$. For a point $a \in B$, we denote by $G_{a}$ the fiber $g^{-1}(a)$.

(S1) The morphism $g$ is dominant.

(S2) Let $\Sigma_{g} \subset B$ be the topological discriminant locus of $g$. Then the Zariski closed subset

$$
\Xi_{2}:=\Sigma_{f} \cap \Sigma_{g}
$$

of $B$ is of codimension $\geq 2$.

(S3) The Zariski closed subset

$$
\Xi_{3}:=\left\{a \in B \mid G_{a} \backslash\left(G_{a} \cap \operatorname{Sing} f\right)=\emptyset\right\}=
$$

of $B$ is of codimension $\geq 2$.

(S4) If $a \in B$ is general, then $G_{a}$ is connected, and the inclusion of $G_{a}$ into $F_{a}$ induces a surjective homomorphism from $\pi_{1}\left(G_{a}\right)$ to $\pi_{1}\left(F_{a}\right)$.

Proposition 4.1. Suppose the following:

(a) The Zariski closed subset

$$
\Xi_{4}:=\left\{a \in B \mid F_{a} \backslash\left(F_{a} \cap \operatorname{Sing} f\right) \text { is empty or not connected }\right\}=
$$

of $B$ is of codimension $\geq 2$. 
(b) The condition (S) is satisfied.

(c) The Zariski closed subset Sing $f$ of $E$ is of codimension $\geq 2$.

Then there exists a homomorphism $\partial$ from $\pi_{2}(B, b)$ to $\pi_{1}\left(F_{b}, \tilde{b}\right)$ such that Ker $i_{*}$ coincides with $\operatorname{Im} \partial$.

Proof. There exists a proper Zariski closed subset $\Sigma_{(f, g)}$ of $B$ containing the union $\Sigma_{f} \cup \Sigma_{g}$ such that the pair

$$
(f, g):(E, S) \rightarrow B
$$

of the morphisms $f$ and $g$ are locally trivial over $B \backslash \Sigma_{(f, g)}$. Let $\Sigma_{(f, g)}^{\prime}$ be the union of all irreducible components of $\Sigma_{(f, g)}$ that are not contained in $\Sigma_{f} \cup \Sigma_{g}$. We put

$$
\Xi_{5}:=\Sigma_{(f, g)}^{\prime} \cap\left(\Sigma_{f} \cup \Sigma_{g}\right)
$$

which is a Zariski closed subset of $B$ with codimension $\geq 2$. The Zariski closed subset

$$
\Xi:=\Xi_{2} \cup \Xi_{3} \cup \Xi_{4} \cup \Xi_{5}
$$

of $B$ is of codimension $\geq 2$ by the assumptions.

Definition 4.2. We say that a continuous map

$$
w:\left(I^{2}, \partial I^{2}\right) \rightarrow(B, b)
$$

is good if $w$ intersects $\Sigma_{(f, g)}$ transversely, and $w^{-1}(\Xi)$ is empty. A lift

$$
\widetilde{w}: I^{2} \rightarrow E
$$

of a good continuous map $w$ is said to be a good lift if $\widetilde{w}(K)$ consists of a single point $\tilde{b}$, where $K:=(I \times\{0\}) \cup(\partial I \times I)$, and $\widetilde{w}^{-1}(\operatorname{Sing} f)$ is empty.

Let $[w]$ be an element of $\pi_{2}(B, b)$. We can assume that $[w]$ is represented by a good continuous map $w$ by Corollary 2.4. Because $\Sigma_{f}$ is contained in $\Sigma_{(f, g)}, w$ intersects $\Sigma_{f}$ transversely. Because $\Xi_{1}$ in Proposition 3.1 is contained in $\Xi_{4}$, we have $w^{-1}\left(\Xi_{1}\right)=\emptyset$. There is a homeomorphism

$$
\zeta:\left(I^{2}, I \times\{0\}\right) \stackrel{\sim}{\rightarrow}\left(I^{2}, K\right)
$$

such that $w \circ \zeta$ intersects $\Sigma_{f}$ transversely. By Lemma 3.3, we can lift $w \circ \zeta$ to

$$
(w \circ \zeta)^{\sim}:\left(I^{2}, I \times\{0\}\right) \rightarrow(E, \tilde{b})
$$

such that $(w \circ \zeta)^{\sim}\left(I^{2}\right) \cap \operatorname{Sing} f=\emptyset$. Putting $\widetilde{w}:=(w \circ \zeta)^{\sim} \circ \zeta^{-1}$, we obtain a good lift

$$
\widetilde{w}:\left(I^{2}, K\right) \rightarrow(E, \tilde{b})
$$

of $w$. We will define $\partial([w])$ to be the element of $\pi_{1}\left(F_{b}, \tilde{b}\right)$ represented by the loop

$$
\widetilde{w} \mid I \times\{1\}:(I, \partial I) \rightarrow\left(F_{b}, \tilde{b}\right) .
$$


To show that this definition makes sense, we will prove that the homotopy class of the loop $\widetilde{w} \mid I \times\{1\}$ in $F_{b}$ does not depend on the choice of a good continuous map $w$ representing $[w]$, and a good lift $\widetilde{w}$ of $w$. Let

$$
w_{0}:\left(I^{2}, \partial I^{2}\right) \rightarrow(B, b) \text { and } w_{1}:\left(I^{2}, \partial I^{2}\right) \rightarrow(B, b)
$$

be good continuous maps representing a same element $[w] \in \pi_{2}(B, b)$, and let

$$
\widetilde{w_{0}}:\left(I^{2}, K\right) \rightarrow(E, \tilde{b}) \text { and } \widetilde{w_{1}}:\left(I^{2}, K\right) \rightarrow(E, \tilde{b})
$$

be good lifts of $w_{0}$ and $w_{1}$, respectively. There exists a homotopy

$$
h:\left(I^{2}, \partial I^{2}\right) \times I \rightarrow(B, b)
$$

from $w_{0}$ to $w_{1}$. We choose a sufficiently small positive real number $\tau$, and let

$$
\rho: I^{2} \times I \rightarrow I^{2} \times I
$$

be the continuous map defined by

$$
\rho(p, t):= \begin{cases}(p, 0) & \text { if } t \in[0,4 \tau], \\ (p,(t-4 \tau) /(1-8 \tau)) & \text { if } t \in[4 \tau, 1-4 \tau], \\ (p, 1) & \text { if } t \in[1-4 \tau, 1] .\end{cases}
$$

Then the continuous map

$$
h \circ \rho:\left(I^{2}, \partial I^{2}\right) \times I \rightarrow(B, b)
$$

is also a homotopy from $w_{0}$ to $w_{1}$. We replace $h$ by $h \circ \rho$. By the definition of good continuous maps, both of $w_{0}^{-1}(\Xi)$ and $w_{1}^{-1}(\Xi)$ are empty. Hence we have

$$
h^{-1}(\Xi) \subset I^{2} \times(4 \tau, 1-4 \tau) .
$$

Moreover, there exist open subsets $A_{0}$ and $A_{1}$ of $I^{2}$ satisfying

$$
w_{\nu}^{-1}\left(\Sigma_{(f, g)}\right) \subset A_{\nu} \subset \overline{A_{\nu}} \subset\left(I^{2}\right)^{\circ} \quad(\nu=0,1)
$$

such that the restrictions

$$
w_{\nu} \mid A_{\nu}: A_{\nu} \rightarrow B \quad(\nu=0,1)
$$

of $w_{\nu}$ to $A_{\nu}$ are $\mathcal{C}^{\infty}$-maps intersecting $\Sigma_{(f, g)}$ transversely. We put

$$
A:=\left(A_{0} \times(0,4 \tau)\right) \cup\left(A_{1} \times(1-4 \tau, 1)\right) .
$$

Then $h \mid A$ is a $\mathcal{C}^{\infty}$-map intersecting $\Sigma_{(f, g)}$ transversely, and $(h \mid A)^{-1}(\Xi)$ is empty. There exist open subsets $V$ and $W$ of $I^{2} \times I$ satisfying

$$
\begin{gathered}
h^{-1}\left(\Sigma_{(f, g)} \cup \Xi\right) \cap\left(I^{2} \times[3 \tau, 1-3 \tau]\right) \subset V \subset \bar{V} \\
\subset W \subset \bar{W} \subset\left(I^{2}\right)^{\circ} \times(2 \tau, 1-2 \tau) .
\end{gathered}
$$

By Corollary 2.3, we obtain a continuous map

$$
h^{\prime}: I^{2} \times I \rightarrow B
$$

with the following properties; 
- $h$ and $h^{\prime}$ are homotopic relative to $\left(I^{2} \times I\right) \backslash W$, and

- the restriction $h^{\prime} \mid A \cup V$ is a $\mathcal{C}^{\infty}$-map intersecting $\Sigma_{(f, g)}$ transversely, and its image is disjoint from $\Xi$.

In particular, $h^{\prime}$ is again a homotopy from $w_{0}$ to $w_{1}$ stationary on $\partial I^{2}$. We replace $h$ by $h^{\prime}$. Then the homotopy $h$ has the following properties;

- $h^{-1}(\Xi)=\emptyset$,

- $h^{-1}\left(\Sigma_{(f, g)}\right)$ is a one-dimensional manifold with the boundary, and the boundary $\partial h^{-1}\left(\Sigma_{(f, g)}\right)$ is contained in $\left(I^{2}\right)^{\circ} \times \partial I$,

- $h(p, t)=h(p, 0)$ if $t \in[0,2 \tau]$, while $h(p, t)=h(p, 1)$ if $t \in[1-2 \tau, 1]$.

Note that $h^{-1}\left(\Sigma_{f}\right), h^{-1}\left(\Sigma_{g}\right)$ and $h^{-1}\left(\Sigma_{(f, g)}^{\prime}\right)$ are disjoint, and each of them is a union of connected components of $h^{-1}\left(\Sigma_{(f, g)}\right)$.

We put

$$
L:=(K \times I) \cup\left(I^{2} \times \partial I\right)
$$

and define a lift

$$
(h \mid L)^{\sim}: L \rightarrow E
$$

of $h \mid L$ by the following;

$$
(h \mid L)^{\sim}(p, t):= \begin{cases}\tilde{b} & \text { if } p \in K, \\ \widetilde{w_{0}}(p) & \text { if } t=0, \\ \widetilde{w_{1}}(p) & \text { if } t=1 .\end{cases}
$$

For the well-definedness of $\partial$, it is enough to show that the lift $(h \mid L) \sim$ extends to a lift

$$
\widetilde{h}:\left(I^{2}, K\right) \times I \rightarrow(E, \tilde{b})
$$

of $h$; that is, there exists a lift $\widetilde{h}$ of $h$ such that $\widetilde{h} \mid L$ coincides with $(h \mid L)^{\sim}$. Because the restriction of such a lift $\widetilde{h}$ to $I \times\{1\} \times I$ will yield a homotopy from the loop $\widetilde{w_{0}} \mid I \times\{1\}$ to the loop $\widetilde{w_{1}} \mid I \times\{1\}$ in $F_{b}$.

We will modify $h$ by a homeomorphism

$$
\Psi: I^{2} \times I \stackrel{\sim}{\rightarrow} I^{2} \times I .
$$

We denote by

$$
H: I^{2} \times I \rightarrow B
$$

the composite $h \circ \Psi$ of $h$ and $\Psi$. We put

$$
\Gamma:=H^{-1}\left(\Sigma_{f}\right) .
$$

For a subset $J$ of $I$, we put

$$
\Gamma_{J}:=\Gamma \cap\left(I^{2} \times J\right) .
$$

When $J$ consists of a single point $t$, we write $\Gamma_{t}$ instead of $\Gamma_{\{t\}}$. By choosing an appropriate homeomorphism $\Psi$, we can assume the following.

( $\Psi 1) \Psi^{-1}(L)=I^{2} \times\{0\}$. 

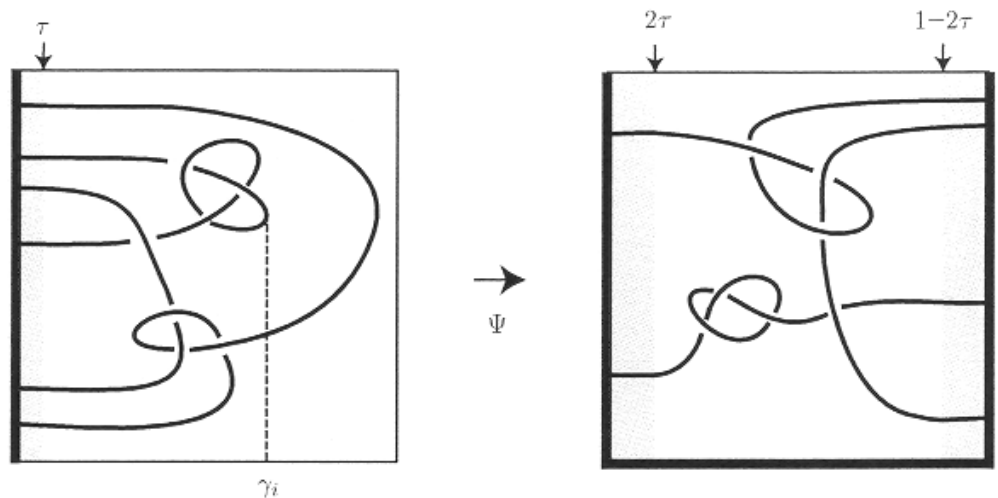

FIG. 4.1. The homeomorphism $\Psi$

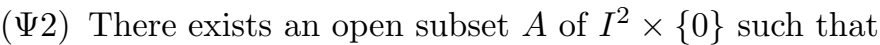

- $\Gamma_{0} \subset A \subset \bar{A} \subset\left(I^{2}\right)^{\circ} \times\{0\}$, and

- $H(p, t)=H(p, 0)$ if $(p, 0) \in A$ and $t \in[0, \tau]$.

In particular, we have $\Gamma_{[0, \tau]}=\Gamma_{0} \times[0, \tau]$.

( $\Psi 3$ ) The closed subset $\Gamma$ of $I^{2} \times I$ is a closed submanifold of $\left(I^{2}\right)^{\circ} \times I$ with boundary. From $(\Psi 1)$ and $(\Psi 2)$ above, the boundary $\partial \Gamma$ is equal to $\Gamma_{0}$, which is a finite subset of points of $A$. The interior $\Gamma^{\circ}=\Gamma \backslash \partial \Gamma$ of $\Gamma$ is a one-dimensional manifold.

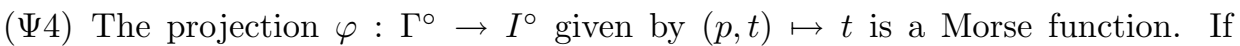
$\left(q_{i}, \gamma_{i}\right) \in \Gamma^{\circ}$ and $\left(q_{j}, \gamma_{j}\right) \in \Gamma^{\circ}$ are distinct critical points of $\varphi$, then the critical values $\gamma_{i}$ and $\gamma_{j}$ are also distinct.

Let $\left(q_{1}, \gamma_{1}\right), \ldots,\left(q_{N}, \gamma_{N}\right)$ be the critical points of $\varphi$. By renumbering, we have

$$
\tau<\gamma_{1}<\cdots<\gamma_{N}<1 .
$$

For $t \in I$, we define

$$
H_{t}: I^{2} \times[0, t] \rightarrow B
$$

to be the restriction of $H$ to $I^{2} \times[0, t]$. We can define a lift

$$
\widetilde{H}_{0}: I^{2} \times\{0\} \rightarrow E
$$

of $H_{0}$ by

$$
\widetilde{H}_{0}:=(h \mid L)^{\sim} \circ\left(\Psi \mid I^{2} \times\{0\}\right),
$$

because $\Psi$ induces a homeomorphism from $I^{2} \times\{0\}$ to $L$ by $(\Psi 1)$ above. It is enough to show that $\widetilde{H}_{0}$ extends to a lift

$$
\widetilde{H}_{1}: I^{2} \times I \rightarrow E
$$

of $H=H_{1}$, because $\widetilde{H}_{1} \circ \Psi^{-1}$ will be the desired lift $\widetilde{h}$ of $h=H \circ \Psi^{-1}$. 
Definition 4.3. We say that a lift $\widetilde{H}_{t}$ of $H_{t}$ has the property $(\mathrm{V})$ if there exists an open subset $V_{t}$ of $I^{2} \times\{t\}$ satisfying

- $\Gamma_{t} \subset V_{t} \subset \bar{V}_{t} \subset\left(I^{2}\right)^{\circ} \times\{t\}$, and

- $\widetilde{H}_{t}$ maps $V_{t}$ to the subvariety $S$ of $E$.

First we will extend $\widetilde{H}_{0}$ to a lift

$$
\widetilde{H}_{\tau}: I^{2} \times[0, \tau] \rightarrow E
$$

of $H_{\tau}$ with the property $(\mathrm{V})$. We put

$$
\Gamma_{0}=\left\{\left(c_{1}, 0\right), \ldots,\left(c_{m}, 0\right)\right\} .
$$

Then we have

$$
H\left(c_{\nu}, t\right)=H\left(c_{\nu}, 0\right) \quad \text { for } \quad t \in[0, \tau] .
$$

We put

$$
a_{\nu}:=H\left(c_{\nu}, 0\right) \in \Sigma_{f}, \quad \text { and } \quad \alpha_{\nu}:=\widetilde{H}_{0}\left(c_{\nu}, 0\right) \in F_{a_{\nu}} .
$$

Because ${\widetilde{w_{0}}}^{-1}(\operatorname{Sing} f)=\emptyset$ and ${\widetilde{w_{1}}}^{-1}(\operatorname{Sing} f)=\emptyset$ by the definition of good lifts, the point $\alpha_{\nu}=(h \mid L)^{\sim}\left(\Psi\left(c_{\nu}, 0\right)\right)$ is not contained in Sing $f$. Because $h^{-1}(\Xi)=\emptyset$, we have $H^{-1}(\Xi)=\emptyset$, and hence $a_{\nu} \notin \Xi$. This implies the following;

- $F_{a_{\nu}} \backslash\left(F_{a_{\nu}} \cap \operatorname{Sing} f\right)$ is connected,

- $G_{a_{\nu}} \not \subset \operatorname{Sing} f$, and

- $a_{\nu} \notin \Sigma_{g}$. In particular, $g$ is smooth at every point of $G_{a_{\nu}}$.

We choose a point $\alpha_{\nu}^{\prime} \in G_{a_{\nu}} \backslash\left(G_{a_{\nu}} \cap \operatorname{Sing} f\right)$. Then we can connect $\alpha_{\nu}$ and $\alpha_{\nu}^{\prime}$ by a path

$$
\xi_{\nu}: I \rightarrow F_{a_{\nu}} \backslash\left(F_{a_{\nu}} \cap \operatorname{Sing} f\right) .
$$

Since $\xi_{\nu}(I)$ is compact, there exist, by Lemma 2.5, an open neighborhood $D_{\nu}$ of $a_{\nu}$ in $B$ and an open neighborhood $M_{\nu}$ of $\xi_{\nu}(I)$ in $f^{-1}\left(D_{\nu}\right)$ such that the pair

$$
\left(f\left|M_{\nu}, g\right| M_{\nu} \cap S\right):\left(M_{\nu}, M_{\nu} \cap S\right) \rightarrow D_{\nu}
$$

is locally trivial. We choose a small closed disk $\Delta_{\nu}$ in $I^{2}$ with the center $c_{\nu}$ such that

- $\Delta_{\nu} \times\{0\} \subset A$, where $A$ is the open subset of $I^{2} \times\{0\}$ that has appeared in $(\Psi 2)$, and

- $\widetilde{H}_{0}(p, 0) \in M_{\nu}$ for all $p \in \Delta_{\nu}$.

Then $H(p, 0) \in D_{\nu}$ for all $p \in \Delta_{\nu}$, and hence we have

$$
H\left(\Delta_{\nu} \times[0, \tau]\right) \subset D_{\nu}
$$

We can define a lift

$$
\left(H \mid\left(\left\{c_{\nu}\right\} \times[0, \tau]\right)\right)^{\sim}:\left\{c_{\nu}\right\} \times[0, \tau] \rightarrow E
$$

of $H \mid\left(\left\{c_{\nu}\right\} \times[0, \tau]\right)$ by

$$
\left(H \mid\left(\left\{c_{\nu}\right\} \times[0, \tau]\right)\right)^{\sim}\left(c_{\nu}, t\right):=\xi_{\nu}(t / \tau) \in F_{a_{\nu}} .
$$


This lift satisfies

$$
\begin{aligned}
& \left(H \mid\left(\left\{c_{\nu}\right\} \times[0, \tau]\right)\right)^{\sim}\left(c_{\nu}, 0\right)=\alpha_{\nu}=\widetilde{H}_{0}\left(c_{\nu}, 0\right), \quad \text { and } \\
& \left(H \mid\left(\left\{c_{\nu}\right\} \times[0, \tau]\right)\right)^{\sim}\left(c_{\nu}, \tau\right)=\alpha_{\nu}^{\prime} \in G_{a_{\nu}} \cap M_{\nu} .
\end{aligned}
$$

Since $g \mid\left(M_{\nu} \cap S\right)$ is locally trivial over $D_{\nu}$, and $H\left(\Delta_{\nu} \times\{\tau\}\right)$ is contained in $D_{\nu}$, we have a lift

$$
\left(H \mid \Delta_{\nu} \times\{\tau\}\right)^{\sim}: \Delta_{\nu} \times\{\tau\} \rightarrow M_{\nu} \cap S
$$

of $H \mid \Delta_{\nu} \times\{\tau\}$ with respect to $g \mid\left(M_{\nu} \cap S\right)$ such that

$$
\left(H \mid \Delta_{\nu} \times\{\tau\}\right)^{\sim}\left(c_{\nu}, \tau\right)=\alpha_{\nu}^{\prime} .
$$

We put

$$
Q_{\nu}:=\left(\Delta_{\nu} \times\{0\}\right) \cup\left(\left\{c_{\nu}\right\} \times[0, \tau]\right) \cup\left(\Delta_{\nu} \times\{\tau\}\right)
$$

By gluing $\widetilde{H}_{0} \mid \Delta_{\nu} \times\{0\},\left(H \mid\left(\left\{c_{\nu}\right\} \times[0, \tau]\right)\right)^{\sim}$ and $\left(H \mid \Delta_{\nu} \times\{\tau\}\right)^{\sim}$ together, we obtain a lift

$$
\left(H \mid Q_{\nu}\right)^{\sim}: Q_{\nu} \rightarrow E
$$

of $H \mid Q_{\nu}$ such that $\left(H \mid Q_{\nu}\right)^{\sim}\left(Q_{\nu}\right) \subset M_{\nu}$. Since $Q_{\nu}$ is a strong deformation retract of $\Delta_{\nu} \times[0, \tau]$, and $\Delta_{\nu} \times[0, \tau]$ is mapped in $D_{\nu}$ by $H$, we can use the homotopy lifting property of the locally trivial map $f \mid M_{\nu}: M_{\nu} \rightarrow D_{\nu}$, and extend $\left(H \mid Q_{\nu}\right)^{\sim}$ to a lift

$$
\left(H \mid \Delta_{\nu} \times[0, \tau]\right)^{\sim}: \Delta_{\nu} \times[0, \tau] \rightarrow E
$$

of $H \mid \Delta_{\nu} \times[0, \tau]$. We then put

$$
R:=\left(I^{2} \times\{0\}\right) \cup \bigcup_{\nu=1}^{m}\left(\Delta_{\nu} \times[0, \tau]\right) .
$$

Gluing $\widetilde{H}_{0}$ and $\left(H \mid \Delta_{\nu} \times[0, \tau]\right)^{\sim}(i=1, \ldots, m)$ together, we obtain a lift

$$
(H \mid R)^{\sim}: R \rightarrow E
$$

of $H \mid R$. Since $R$ is a strong deformation retract of $I^{2} \times[0, \tau]$, and $f$ is locally trivial over

$$
H \mid\left(\left(I^{2} \times[0, \tau]\right) \backslash R^{\circ}\right):\left(I^{2} \times[0, \tau]\right) \backslash R^{\circ} \rightarrow B,
$$

we can extend $(H \mid R)^{\sim}$ to a lift

$$
\widetilde{H}_{\tau}: I^{2} \times[0, \tau] \rightarrow E
$$

of $H_{\tau}$. By the construction, $\widetilde{H}_{\tau}$ is an extension of $\widetilde{H}_{0}$. Moreover, since $\cup_{\nu}\left(\Delta_{\nu}^{\circ} \times\right.$ $\{\tau\})$ is an open subset of $I^{2} \times\{\tau\}$ containing $\Gamma_{\tau}$ and is mapped into $\cup_{\nu}\left(M_{\nu} \cap S\right) \subset S$ by $\widetilde{H}_{\tau}$, this extension $\widetilde{H}_{\tau}$ has the property $(\mathrm{V})$.

Next we prove the following:

Claim 4.4. Suppose that we are given a closed interval $[u, v]$ contained in $[\tau, 1]$ and a lift

$$
\widetilde{H}_{u}: I^{2} \times[0, u] \rightarrow E
$$




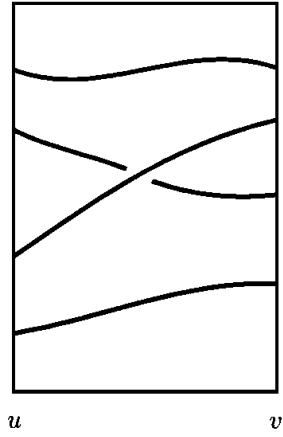

Case 1

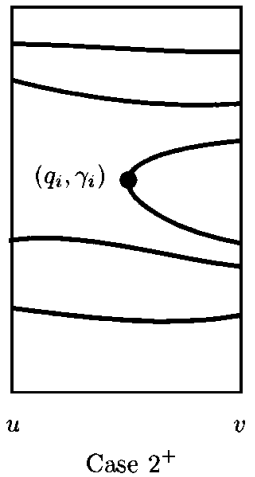

Case $2^{+}$

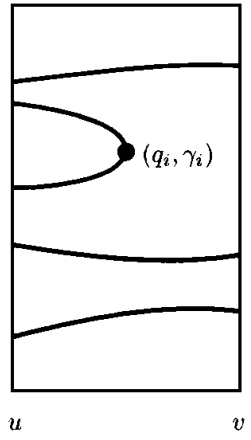

Case $2^{-}$

FIG. 4.2. The three cases

of $H_{u}$ with the property $(\mathrm{V})$. Then $\widetilde{H}_{u}$ can be extended to a lift

$$
\widetilde{H}_{v}: I^{2} \times[0, v] \rightarrow E
$$

of $H_{v}$ with the property $(\mathrm{V})$ in the following two cases:

Case 1. The closed interval $[u, v]$ contains no critical values of the Morse function $\varphi: \Gamma^{\circ} \rightarrow I^{\circ}$. (See the condition $(\Psi 4)$.)

Case 2. There exists a critical value $\gamma_{i}$ of $\varphi$ such that $u=\gamma_{i}-\varepsilon$ and $v=\gamma_{i}+\varepsilon$, where $\varepsilon$ is a sufficiently small positive real number.

Starting from $\widetilde{H}_{\tau}$ and repeating the extension of $\widetilde{H}_{u}$ to $\widetilde{H}_{v}$ in the above two cases, we can extend $\widetilde{H}_{\tau}$ to a lift $\widetilde{H}_{1}: I^{2} \times I \rightarrow I$ of $H=H_{1}$. The well-definedness of the boundary homomorphism $\partial: \pi_{2}(B) \rightarrow \pi_{2}\left(F_{b}\right)$ will thus be established.

Proof of Claim 4.4. We divide Case 2 into the following two sub-cases:

Case $2^{+}$. The critical point $\left(q_{i}, \gamma_{i}\right)$ of $\varphi$ is of index +1 .

Case $2^{-}$. The critical point $\left(q_{i}, \gamma_{i}\right)$ of $\varphi$ is of index -1 .

By the property $(\mathrm{V})$ of $\widetilde{H}_{u}$, we have an open subset $V\left(\Gamma_{u}\right)$ of $I^{2} \times\{u\}$ such that

$$
\Gamma_{u} \subset V\left(\Gamma_{u}\right) \subset \overline{V\left(\Gamma_{u}\right)} \subset\left(I^{2}\right)^{\circ} \times\{u\},
$$

and that $\widetilde{H}_{u}$ maps $V\left(\Gamma_{u}\right)$ into $S$. We decompose $\Gamma_{[u, v]}$ into the disjoint union of $\Gamma_{[u, v]}^{\prime}$ and $\Gamma_{[u, v]}^{\prime \prime}$, where $\Gamma_{[u, v]}^{\prime \prime}$ is the union of all connected components of $\Gamma_{[u, v]}$ that do not contain any critical point of $\varphi$, and $\Gamma_{[u, v]}^{\prime}:=\Gamma_{[u, v]} \backslash \Gamma_{[u, v]}^{\prime \prime}$. In Case 1 , $\Gamma_{[u, v]}^{\prime}$ is empty, while in Case 2, $\Gamma_{[u, v]}^{\prime}$ consists of a single connected component. In both cases, each connected component of $\Gamma_{[u, v]}^{\prime \prime}$ is mapped homeomorphically onto $[u, v]$ by the projection $\varphi$. We put

$$
\Gamma_{u}^{\prime \prime}:=\Gamma_{[u, v]}^{\prime \prime} \cap\left(I^{2} \times\{u\}\right)=\left\{\left(c_{1}, u\right), \ldots,\left(c_{k}, u\right)\right\} .
$$


There exists a trivialization

$$
\left(I^{2} \times[u, v], \Gamma_{[u, v]}^{\prime \prime}\right) \backslash \underset{[u, v]}{\stackrel{\sim}{\sim}} \swarrow \quad\left(I^{2} \times\{u\}, \Gamma_{u}^{\prime \prime}\right) \times[u, v]
$$

of the pair $\left(I^{2} \times[u, v], \Gamma_{[u, v]}^{\prime \prime}\right)$ of topological spaces over $[u, v]$ such that the restriction $\sigma \mid I^{2} \times\{u\}$ of the homeomorphism $\sigma$ is the identity map of $I^{2} \times\{u\}$. For each point $\left(c_{\nu}, u\right)$ of $\Gamma_{u}^{\prime \prime}$, we choose a sufficiently small closed disk $\Delta_{\nu}$ in $I^{2}$ with the center $c_{\nu}$ such that $\Delta_{\nu} \times\{u\} \subset V\left(\Gamma_{u}\right)$, and put

$$
T_{\nu}:=\sigma^{-1}\left(\Delta_{\nu} \times[u, v]\right), \quad T:=\bigcup_{\nu=1}^{k} T_{\nu} .
$$

Then $T$ is a closed tubular neighborhood of $\Gamma_{[u, v]}^{\prime \prime}$ in $I^{2} \times[u, v]$. Since we have $H^{-1}(\Xi)=\emptyset, \Gamma \cap H^{-1}\left(\Sigma_{g}\right)$ is empty. Hence, by taking $\Delta_{\nu}$ small enough, we can assume that

$$
T \cap H^{-1}\left(\Sigma_{g}\right)=\emptyset .
$$

Then $g$ is locally trivial over $H \mid T$. Since

$$
T_{u}:=T \cap\left(I^{2} \times\{u\}\right)=\left(\cup \Delta_{\nu}\right) \times\{u\}
$$

is contained in $V\left(\Gamma_{u}\right)$, the property $(\mathrm{V})$ of $\widetilde{H}_{u}$ implies

$$
\widetilde{H}_{u}\left(T_{u}\right) \subset S .
$$

Therefore, using the homotopy lifting property of the pull-back of $g: S \rightarrow B$ by $H \mid T: T \rightarrow B$, we can extend $\widetilde{H}_{u} \mid T_{u}$ to a lift

$$
(H \mid T)^{\sim}: T \rightarrow S
$$

of $H \mid T$ with respect to $g$. Gluing $\widetilde{H}_{u}$ and $(H \mid T)^{\sim}$ together, we obtain a lift

$$
\widetilde{H}_{u, T}:\left(I^{2} \times[0, u]\right) \cup T \rightarrow E
$$

of the restriction

$$
H \mid\left(\left(I^{2} \times[0, u]\right) \cup T\right):\left(I^{2} \times[0, u]\right) \cup T \rightarrow B
$$

of $H$ that satisfies

$$
\widetilde{H}_{u, T}(T) \subset S
$$

Case 1. In this case, we have $\Gamma_{[u, v]}=\Gamma_{[u, v]}^{\prime \prime}$, and hence

$$
\left(I^{2} \times[0, v]\right) \backslash\left(\left(I^{2} \times[0, u]\right) \cup T\right)^{\circ}
$$

is disjoint from $\Gamma$. Hence $f$ is locally trivial over the restriction of $H$ to (4.2). Moreover, $\left(I^{2} \times[0, u]\right) \cup T$ is a strong deformation retract of $I^{2} \times[0, v]$. Hence we can extend $\widetilde{H}_{u, T}$ to a lift

$$
\widetilde{H}_{v}: I^{2} \times[0, v] \rightarrow E
$$




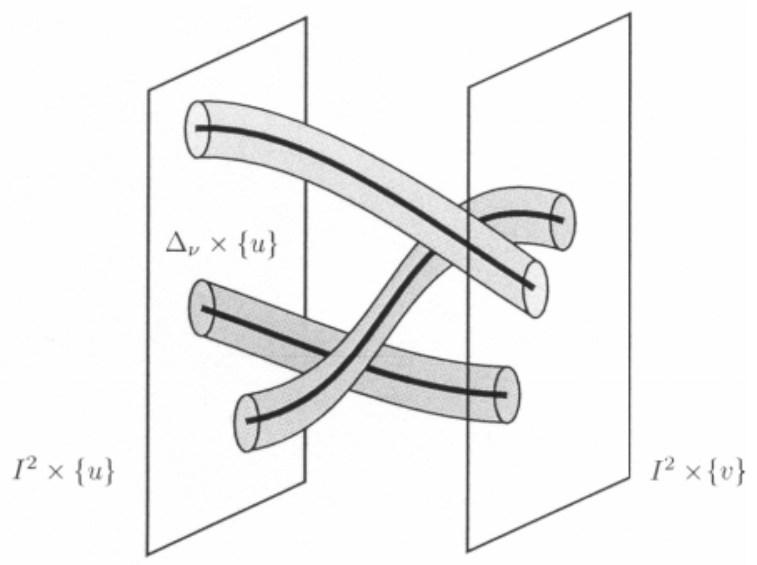

FIG. 4.3. Case 1

of $H_{v}$. Since

$$
T_{v}^{\circ}:=T^{\circ} \cap\left(I^{2} \times\{v\}\right)
$$

is an open subset of $I^{2} \times\{v\}$ containing $\Gamma_{v}$, and $\widetilde{H}_{v}\left(T_{v}^{\circ}\right)=\widetilde{H}_{u, T}\left(T_{v}^{\circ}\right)$ is contained in $S$, the lift $\widetilde{H}_{v}$ thus constructed has the property (V).

Case 2. Note that the critical point $\left(q_{i}, \gamma_{i}\right)$ of the Morse function $\varphi$ in the region $I^{2} \times[u, v]$ is not contained in $H^{-1}\left(\Sigma_{g}\right)$, because $H^{-1}(\Xi)=\emptyset$. Note also that, taking the closed discs $\Delta_{\nu}$ small enough, we can assume that

$$
T \cap \Gamma_{[u, v]}^{\prime}=\emptyset .
$$

Case $\mathbf{2}^{+}$. Since $\varepsilon$ is small enough, there exists a positive real number $\delta$ such that the closed subset

$$
R:=\left\{(p, t) \in I^{2} \times[u, v]|| p-q_{i} \mid \leq \delta, u+\varepsilon / 2 \leq t \leq v\right\}
$$

of $I^{2} \times I$ satisfies the following;

$$
\Gamma_{[u, v]}^{\prime} \subset R, \quad T \cap R=\emptyset \quad \text { and } \quad H^{-1}\left(\Sigma_{g}\right) \cap R=\emptyset .
$$

We put

$$
\Lambda:=\left\{q_{i}\right\} \times[u, u+\varepsilon / 2],
$$

which is a line segment connecting

$$
r_{0}:=\left(q_{i}, u\right) \in I^{2} \times\{u\} \quad \text { and } \quad r_{1}:=\left(q_{i}, u+\varepsilon / 2\right) \in R .
$$

Since $\varepsilon$ is small enough, we can assume that

$$
T \cap \Lambda=\emptyset .
$$

We put

$$
\beta_{0}:=\widetilde{H}_{u}\left(r_{0}\right) \in F_{H\left(r_{0}\right)} .
$$




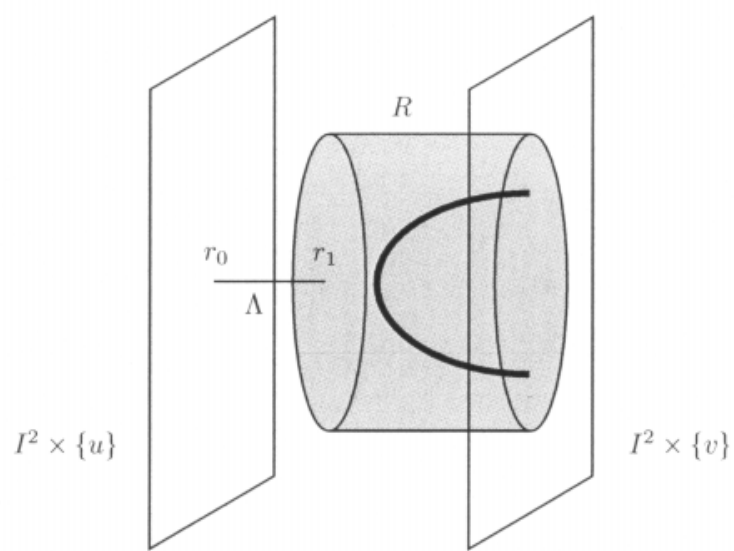

FIG. 4.4. Case $2^{+}$

Since $H\left(r_{1}\right) \notin \Sigma_{g}$, we have a point

$$
\beta_{1} \in G_{H\left(r_{1}\right)} .
$$

Since $H^{-1}\left(\Sigma_{f}\right) \cap \Lambda=\emptyset$, Lemma 3.2 gives us a lift

$$
(H \mid \Lambda)^{\sim}: \Lambda \rightarrow E
$$

of $H \mid \Lambda$ such that

$$
(H \mid \Lambda)^{\sim}\left(r_{0}\right)=\beta_{0}=\widetilde{H}_{u}\left(r_{0}\right) \text { and }(H \mid \Lambda)^{\sim}\left(r_{1}\right)=\beta_{1} \in S .
$$

Since $g$ is locally trivial over $H \mid R$ and $\left\{r_{1}\right\}$ is a strong deformation retract of $R$, we have a lift

$$
(H \mid R)^{\sim}: R \rightarrow S
$$

of $H \mid R$ with respect to $g$ such that

$$
(H \mid R)^{\sim}\left(r_{1}\right)=\beta_{1}=(H \mid \Lambda)^{\sim}\left(r_{1}\right) .
$$

We put

$$
Q:=\left(I^{2} \times[0, u]\right) \cup T \cup \Lambda \cup R .
$$

Gluing $\widetilde{H}_{u, T},(H \mid \Lambda)^{\sim}$ and $(H \mid R)^{\sim}$ together, we obtain a lift

$$
(H \mid Q)^{\sim}: Q \rightarrow E
$$

of $H \mid Q$. Since the interior of $Q$ contains $\Gamma_{[0, v]}, f$ is locally trivial over

$$
H \mid\left(\left(I^{2} \times[0, v]\right) \backslash Q^{\circ}\right):\left(I^{2} \times[0, v]\right) \backslash Q^{\circ} \rightarrow B .
$$

Moreover, $Q$ is a strong deformation retract of $I^{2} \times[0, v]$. Hence we can extend $(H \mid Q)^{\sim}$ to a lift

$$
\widetilde{H}_{v}: I^{2} \times[0, v] \rightarrow E
$$


of $H_{v}$. Note that

$$
\left(R^{\circ} \cup T^{\circ}\right) \cap\left(I^{2} \times\{v\}\right)
$$

is an open subset of $I^{2} \times\{v\}$ containing $\Gamma_{v}$, and it is mapped to $S$ by $\widetilde{H}_{v}$ from the construction. Thus the lift $\widetilde{H}_{v}$ has the property $(\mathrm{V})$.

Case $2^{-}$. Since $H^{-1}(\Xi)=\emptyset$, we have

$$
H\left(q_{i}, \gamma_{i}\right) \notin \Sigma_{g} \cup \Sigma_{(f, g)}^{\prime} .
$$

Since $\varepsilon$ is small enough, there exist an open ball $V$ in $\left(I^{2} \times I\right)^{\circ}$ containing $\left(q_{i}, \gamma_{i}\right)$ and coordinates $(x, y, z)$ defined on $V$ such that the following hold:

- $V \supset \Gamma_{[u, v]}^{\prime}, V \cap H^{-1}\left(\Sigma_{g}\right)=\emptyset, V \cap H^{-1}\left(\Sigma_{(f, g)}^{\prime}\right)=\emptyset, V \cap T=\emptyset$.

- The critical point $\left(q_{i}, \gamma_{i}\right)$ is the origin $(0,0,0)$ of the coordinates $(x, y, z)$.

- The projection $V \rightarrow I$ onto the second factor of $I^{2} \times I$ is given by

$$
(x, y, z) \mapsto z+\gamma_{i}
$$

- The real one-dimensional curve $\Gamma_{[u, v]}^{\prime}$ is given by

$$
\left\{\left(0, s, \gamma_{i}-s^{2}\right) \mid-\sqrt{\varepsilon} \leq s \leq \sqrt{\varepsilon}\right\}
$$

in terms of $(x, y, z)$.

We introduce the usual Euclidean distance $\|\cdot, \cdot\|$ on $V$ with respect to $(x, y, z)$. Let $\rho$ be a sufficiently small positive real number, and let $P$ be the set of all $(p, t) \in V$ satisfying

- $t \in[u, v]$, and

- there exists a point $\left(p^{\prime}, t^{\prime}\right) \in \Gamma_{[u, v]}^{\prime}$ such that $\left\|(p, t),\left(p^{\prime}, t^{\prime}\right)\right\| \leq \rho$.

Then $P$ is a closed tubular neighborhood of $\Gamma_{[u, v]}^{\prime}$ in $I^{2} \times[u, v]$. Taking $\rho$ small enough, we can assume that

$$
P_{u}:=P \cap\left(I^{2} \times\{u\}\right)
$$

is contained in the open neighborhood $V\left(\Gamma_{u}\right)$ of $\Gamma_{u}$ in $I^{2} \times\{u\}$. Let $D$ be the connected component of

$$
\left(\left(V \cap\left(I^{2} \times[u, v]\right)\right) \backslash P^{\circ}\right) \cap\{x=0\}
$$

containing the point $(0,0,-\varepsilon)$. Then $D$ is homeomorphic to the 2-dimensional closed disk. We put

$$
\Lambda_{0}:=D \cap\left(I^{2} \times\{u\}\right), \quad \text { and } \quad \Lambda_{1}:=D \cap P .
$$

Let $r_{0}$ and $r_{1}$ be the end-points of the line segment $\Lambda_{0}$. Then $\Lambda_{1}$ is a curve segment on the boundary of the tube $P$ connecting $r_{0}$ and $r_{1}$, and the boundary $\partial D$ of the disk $D$ is $\Lambda_{0} \cup \Lambda_{1}$. Since

$$
H^{-1}\left(\Sigma_{(f, g)}\right) \cap D=\left(\Gamma \cup H^{-1}\left(\Sigma_{g}\right) \cup H^{-1}\left(\Sigma_{(f, g)}^{\prime}\right)\right) \cap D=\emptyset,
$$




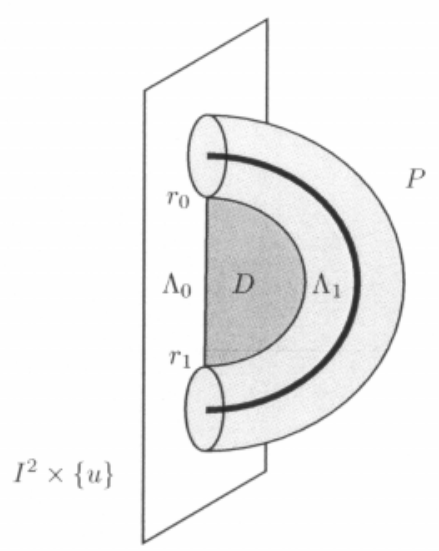

Fig. 4.5. Case $2^{-}$

the pair $(f, g)$ of the morphisms is locally trivial over $H \mid D$. Since $D$ is contractible, we have a trivialization

$$
\begin{aligned}
& \left((H \mid D)^{*} E,(H \mid D)^{*} S\right) \underset{\psi_{D}}{\stackrel{\sim}{\sim}}(F, G) \times D \\
& (H \mid D)^{*}(f, g) \searrow \quad \swarrow \operatorname{pr}_{D} \\
& \text { D }
\end{aligned}
$$

of the pull-back of $(f, g)$ by $H \mid D$, where $(F, G)$ is a pair of topological spaces homeomorphic to the general fiber of $(f, g)$. The continuous map

$$
\widetilde{H}_{u} \mid \Lambda_{0}: \Lambda_{0} \rightarrow E
$$

naturally yields a lift

$$
s_{0}: \Lambda_{0} \rightarrow(H \mid D)^{*} E
$$

of $\Lambda_{0} \hookrightarrow D$ with respect to $(H \mid D)^{*} f$. We fix a homeomorphic path

$$
\lambda: I \rightarrow \Lambda_{0}
$$

from $r_{0}$ to $r_{1}$, and define a path $s_{0}^{\prime}: I \rightarrow F$ by

$$
s_{0}^{\prime}(t):=\operatorname{pr}_{F}\left(\psi_{D}\left(s_{0}(\lambda(t))\right)\right),
$$

where

$$
\operatorname{pr}_{F}: F \times D \rightarrow F
$$

is the natural projection. Because $r_{0}$ and $r_{1}$ are contained in $P_{u} \subset V\left(\Gamma_{u}\right), \widetilde{H}_{u}\left(r_{0}\right)$ and $\widetilde{H}_{u}\left(r_{1}\right)$ are contained in $S$. Hence we have

$$
s_{0}^{\prime}(0) \in G, \quad s_{0}^{\prime}(1) \in G .
$$


Note that both of $F$ and $G$ are path-connected. Moreover, from the item (S4) of the condition (S), the inclusion $G \hookrightarrow F$ induces a surjective homomorphism from $\pi_{1}(G)$ to $\pi_{1}(F)$. Since $G$ is path-connected, we have a path

$$
s_{2}^{\prime}: I \rightarrow G
$$

from $s_{0}^{\prime}(1)$ to $s_{0}^{\prime}(0)$. Then $s_{0}^{\prime} s_{2}^{\prime}$ is a loop in $F$ with the base point $s_{0}^{\prime}(0)$. Hence there exists a loop

$$
s_{02}^{\prime}:(I, \partial I) \rightarrow\left(G, s_{0}^{\prime}(0)\right)
$$

such that

$$
\left[s_{02}^{\prime}\right]=\left[s_{0}^{\prime} s_{2}^{\prime}\right] \quad \text { in } \quad \pi_{1}\left(F, s_{0}^{\prime}(0)\right) \text {. }
$$

We define a path

$$
s_{1}^{\prime}: I \rightarrow G
$$

from $s_{0}^{\prime}(0)$ to $s_{0}^{\prime}(1)$ to be the conjunction of the loop $s_{02}^{\prime}$ and the inverse path $\left(s_{2}^{\prime}\right)^{-1}$ of $s_{2}^{\prime}$ in $G$;

$$
s_{1}^{\prime}:=s_{02}^{\prime} \cdot\left(s_{2}^{\prime}\right)^{-1} \text {. }
$$

Then $s_{0}^{\prime}$ and $s_{1}^{\prime}$ are homotopic in $F$ relative to $\partial I$. Thus we have a homotopy

$$
\eta: I \times I \rightarrow F
$$

from $s_{0}^{\prime}$ to $s_{1}^{\prime}$ stationary on $\partial I$. We have a continuous map

$$
\tau: I \times I \rightarrow D
$$

such that

- $\tau$ maps $I \times\{0\}$ to $\Lambda_{0}$ and $I \times\{1\}$ to $\Lambda_{1}$ homeomorphically,

- $\tau$ contracts $\{0\} \times I$ to the point $r_{0}$, and $\{1\} \times I$ to the point $r_{1}$,

- the homeomorphism $\tau \mid I \times\{0\}$ from $I \times\{0\}$ to $\Lambda_{0}$ coincides with the homeomorphic path $\lambda: I \rightarrow \Lambda_{0}$, and

- $\tau$ induces a homeomorphism from $I^{\circ} \times I$ to $D \backslash\left\{r_{0}, r_{1}\right\}$.

Then there exists a unique continuous map

$$
\eta_{D}: D \rightarrow F
$$

such that $\eta$ factors as $\eta_{D} \circ \tau$. We define a continuous map

$$
\widetilde{\eta}_{D}: D \rightarrow F \times D
$$

by $\widetilde{\eta}_{D}(p):=\left(\eta_{D}(p), p\right)$. Then we can define a lift

$$
(H \mid D)^{\sim}: D \rightarrow E
$$

of $H \mid D$ by

$$
(H \mid D)^{\sim}:=\operatorname{pr}_{E} \circ \psi_{D}^{-1} \circ \widetilde{\eta}_{D}
$$

where $\operatorname{pr}_{E}:(H \mid D)^{*} E \rightarrow E$ is the natural projection, and $\psi_{D}$ is the homeomorphism from $(H \mid D)^{*} E$ to $F \times D$ that has appeared in the trivialization of $(H \mid D)^{*}(f, g)$ over $D$. Then we have

$$
(H \mid D)^{\sim}\left|\Lambda_{0}=\widetilde{H}_{u}\right| \Lambda_{0}
$$


because $(H \mid D)^{\sim} \mid \Lambda_{0}$ coincides with

$$
\operatorname{pr}_{E} \circ s_{0}: \Lambda_{0} \rightarrow E
$$

by the construction. Hence we can glue $\widetilde{H}_{u, T}$ and $(H \mid D)^{\sim}$ along $\Lambda_{0}$, and obtain a lift

$$
\widetilde{H}_{u, T \cup D}:\left(I^{2} \times[0, u]\right) \cup T \cup D \rightarrow E
$$

of $H \mid\left(\left(I^{2} \times[0, u]\right) \cup T \cup D\right)$. Since $s_{1}^{\prime}$ is a path in $G$, we have

$$
\widetilde{H}_{u, T \cup D}\left(\Lambda_{1}\right) \subset S \text {. }
$$

Since $P_{u}=P \cap\left(I^{2} \times\{u\}\right)$ is contained in $V\left(\Gamma_{u}\right)$, we have

$$
\widetilde{H}_{u, T \cup D}\left(P_{u}\right) \subset S .
$$

Since $P_{u} \cup \Lambda_{1}$ is a strong deformation retract of $P$, and $g$ is locally trivial over $H \mid P$, we can extend $\widetilde{H}_{u, T \cup D} \mid\left(P_{u} \cup \Lambda_{1}\right)$ to a lift

$$
(H \mid P)^{\sim}: P \rightarrow S
$$

of $H \mid P$ with respect to $g$. We put

$$
R:=\left(I^{2} \times[0, u]\right) \cup T \cup D \cup P .
$$

Gluing $\widetilde{H}_{u, T \cup D}$ and $(H \mid P)^{\sim}$ together, we obtain a lift

$$
(H \mid R)^{\sim}: R \rightarrow E
$$

of $H \mid R$. Since $R$ is a strong deformation retract of $I^{2} \times[0, v]$, and $f$ is locally trivial over $\left(I^{2} \times[0, v]\right) \backslash R^{\circ}$, we can extend $(H \mid R)^{\sim}$ to a lift

$$
\widetilde{H}_{v}: I^{2} \times[0, v] \rightarrow E
$$

of $H_{v}$. Since $T_{v}^{\circ}=T^{\circ} \cap\left(I^{2} \times\{v\}\right)$ is an open subset of $I^{2} \times\{v\}$ containing $\Gamma_{v}$, and

$$
\widetilde{H}_{v}\left(T_{v}^{\circ}\right)=\widetilde{H}_{u, T}\left(T_{v}^{\circ}\right) \subset S,
$$

the lift $\widetilde{H}_{v}$ thus constructed have the property $(\mathrm{V})$.

Thus the proof of Claim 4.4 is completed.

The boundary map $\partial: \pi_{2}(B, b) \rightarrow \pi_{1}\left(F_{b}, \tilde{b}\right)$ is now well-defined. If

$$
w:\left(I^{2}, \partial I^{2}\right) \rightarrow(B, b) \text { and } w^{\prime}:\left(I^{2}, \partial I^{2}\right) \rightarrow(B, b)
$$

are good continuous maps, and

$$
\widetilde{w}:\left(I^{2}, K\right) \rightarrow(E, \tilde{b}) \text { and } \quad \widetilde{w}^{\prime}:\left(I^{2}, K\right) \rightarrow(E, \tilde{b})
$$

are their good lifts, then the continuous map

$$
w+w^{\prime}:\left(I^{2}, \partial I^{2}\right) \rightarrow(B, b)
$$


defined by

$$
\left(w+w^{\prime}\right)(s, t):= \begin{cases}w(2 s, t) & \text { if } s \in[0,1 / 2] \\ w^{\prime}(2 s-1, t) & \text { if } s \in[1 / 2,1]\end{cases}
$$

is also a good continuous map, and the continuous map

$$
\widetilde{w}+\widetilde{w}^{\prime}:\left(I^{2}, K\right) \rightarrow(E, \tilde{b})
$$

defined in the same way is a good lift of $w+w^{\prime}$. Hence $\partial$ is a homomorphism. Now we will prove that $\operatorname{Im} \partial$ coincides with $\operatorname{Ker} i_{*}$, using the condition (c). It is obvious that $\operatorname{Im} \partial$ is contained in $\operatorname{Ker} i_{*}$. Suppose that a loop

$$
v:(I, \partial I) \rightarrow\left(F_{b}, \tilde{b}\right)
$$

represents an element of $\operatorname{Ker} i_{*}$. Then there exists a homotopy

$$
\widetilde{w}:(I, \partial I) \times I \rightarrow(E, \tilde{b})
$$

in $E$ from the constant loop $0_{\tilde{b}}$ to $v$. Note that $\widetilde{w}(K)$ consists of a single point $\tilde{b}$. If $f^{-1}(\Xi)$ had an irreducible component of codimension 1 in $E$, then the assumption that the codimension of $\Xi$ in $B$ is $\geq 2$ implies that this irreducible component of $f^{-1}(\Xi)$ would be contained in $\operatorname{Sing} f$. Hence $f^{-1}(\Xi)$ must be of codimension $\geq 2$ in E. By Corollary 2.4, we can assume that

$$
\widetilde{w}^{-1}(\operatorname{Sing} f)=\emptyset, \quad \widetilde{w}^{-1}\left(f^{-1}(\Xi)\right)=\emptyset,
$$

and that $\widetilde{w}$ intersects $f^{-1}\left(\Sigma_{(f, g)}\right)$ transversely. Then

$$
w:=f \circ \widetilde{w}:\left(I^{2}, \partial I^{2}\right) \rightarrow(B, b)
$$

is a good continuous map, and $\widetilde{w}$ is a good lift of $w$. Hence we have $[v]=\partial([w])$. Therefore $\operatorname{Ker} i_{*}$ is contained in $\operatorname{Im} \partial$.

\section{Proof of Theorem 1.1}

By Propositions 3.1 and 4.1, it is enough to show that the three conditions (C1)(C3) in Theorem 1.1 imply the conditions (a)-(c) in Proposition 4.1. (Note that $\Xi_{1}$ is contained in $\Xi_{4}$.) Since Theorem 1.1 is trivial when $\operatorname{dim} \bar{E}=\operatorname{dim} B$ or $\operatorname{dim} B=0$, we will assume

$$
\operatorname{dim} \bar{E}>\operatorname{dim} B>0 \text {. }
$$

The condition (c) follows immediately from (C2).

Claim 5.1. The Zariski closed subset

$$
\Xi_{6}:=\left\{a \in B \mid \bar{F}_{a} \text { is not irreducible }\right\}=
$$

of $B$ is of codimension $\geq 2$. 
Proof of Claim 5.1. Suppose that $\Xi_{6}$ had an irreducible component $\Xi_{6}^{\prime}$ of codimension 1 in $B$. Let $\xi$ be a general point of $\Xi_{6}^{\prime}$. Since $\bar{F}_{\xi}$ is connected, there exist two irreducible components of $\bar{F}_{\xi}$ intersecting at a point $p$. Since $\xi$ is general in $\Xi_{6}^{\prime}$, $\bar{f}^{-1}\left(\Xi_{6}^{\prime}\right)$ is not locally irreducible at $p$. Let $A_{1}$ and $A_{2}$ be distinct local irreducible components of $\bar{f}^{-1}\left(\Xi_{6}^{\prime}\right)$ at $p$. Then $A_{1} \cap A_{2}$ is of codimension 2 in $\bar{E}$, because both of $A_{1}$ and $A_{2}$ are hypersurfaces in the smooth variety $\bar{E}$. Since $A_{1} \cap A_{2}$ is contained in $\operatorname{Sing} f$, we get a contradiction to (C2).

Claim 5.2. The Zariski closed subset $\Xi_{4}$ of $B$ is of codimension $\geq 2$.

Proof of Claim 5.2. Suppose that $\Xi_{4}$ had an irreducible component $\Xi_{4}^{\prime}$ of codimension 1 in $B$. Let $\xi$ be a general point of $\Xi_{4}^{\prime}$. By Claim 5.1, $\bar{F}_{\xi}$ is irreducible. If $F_{\xi}$ is empty, then $\bar{F}_{\xi}$ is contained in $Z$. Since $\xi$ is general in $\Xi_{4}^{\prime}$, the hypersurface $\bar{f}^{-1}\left(\Xi_{4}^{\prime}\right)$ would be contained in $Z$, and hence we get a contradiction to (C1). If $F_{\xi}$ is non-empty and contained in $\operatorname{Sing} f$, then $\bar{F}_{\xi}$ is contained in Sing $\bar{f}$ and hence we get a contradiction to (C3). Therefore $F_{\xi} \backslash\left(F_{\xi} \cap \operatorname{Sing} f\right)$ is non-empty. Since $\xi \in \Xi_{4}$, the Zariski open subset $F_{\xi} \backslash\left(F_{\xi} \cap \operatorname{Sing} f\right)$ of $\bar{F}_{\xi}$ must be not connected, which contradicts to the irreducibility of $\bar{F}_{\xi}$.

Thus all we have to prove is that the condition $(\mathrm{S})$ is satisfied. Let $\Sigma_{f}^{(1)}, \ldots$, $\Sigma_{f}^{(k)}$ be the irreducible components of $\Sigma_{f}$ with codimension 1 in $B$, and let $\zeta(i)$ be a general point of $\Sigma_{f}^{(i)}$. By Claim 5.1, $\bar{F}_{\zeta(i)}$ is irreducible. By the condition $(\mathrm{C} 2), \bar{F}_{\zeta(i)} \cap \operatorname{Sing} \bar{f}$ is of codimension $\geq 2$ in $\bar{F}_{\zeta(i)}$. By the condition (C3), $Z_{\zeta(i)}$ is reduced.

Since $\bar{E}$ is quasi-projective, we can embed $\bar{E}$ into a projective space $\mathbb{P}^{N}$. We choose a general linear subspace $\Lambda$ of $\mathbb{P}^{N}$ with

$$
\operatorname{dim} \Lambda:=N-\operatorname{dim} \bar{E}+\operatorname{dim} B+1 .
$$

Let $a$ be a general point of $B$, and let $d$ be the degree of $Z_{a}$ in $\mathbb{P}^{N}$. Then $\bar{F}_{a} \cap \Lambda$ is a smooth connected projective curve, and $Z_{a} \cap \Lambda$ consists of distinct $d$ points. Moreover, by Lemma 2.7, the inclusion $F_{a} \cap \Lambda \hookrightarrow F_{a}$ induces a surjective homomorphism from the fundamental group of the punctured Riemann surface $F_{a} \cap \Lambda$ to the fundamental group of $F_{a}$. Moreover $\bar{F}_{\zeta(i)} \cap \Lambda$ is a smooth connected projective curve disjoint from Sing $\bar{f}$ with genus equal to the genus of $\bar{F}_{a} \cap \Lambda$ by Lemma 2.7, and $Z_{\zeta(i)} \cap \Lambda$ consists of distinct $d$ points.

We put

$$
S:=\Lambda \cap E
$$

Then the general fiber $G_{a}$ of $g$ is a compact Riemann surface minus $d$ distinct points, and its fundamental group is mapped surjectively onto the fundamental group of $F_{a}$. Since the genus of the compactification of the fibers of $g$ and the number of the punctured points do not vary locally around $\zeta(i)$, the point $\zeta(i)$ is not contained in $\Sigma_{g}$. Hence $\Xi_{2}=\Sigma_{f} \cap \Sigma_{g}$ is of codimension $\geq 2$ in $B$. 
Let $Z^{(1)}, \ldots, Z^{(n)}$ be the irreducible components of $Z$. Note that

$$
\operatorname{dim} Z^{(j)}=\operatorname{dim} \bar{E}-1>N-\operatorname{dim} \Lambda \text {, }
$$

because we have assumed $\operatorname{dim} B>0$. Since $\Lambda$ is general, Lemma 2.7 implies that $Z^{(j)} \cap \Lambda$ is irreducible of dimension equal to $\operatorname{dim} B$, and the restriction of $\bar{f}$ to $Z^{(j)} \cap \Lambda$ is generically finite. Therefore the locus

$$
\left\{a \in B \mid \operatorname{dim}\left(Z_{a} \cap \Lambda\right) \geq 1\right\}
$$

is contained in a Zariski closed subset of $B$ with codimension $\geq 2$. Hence the locus $\left\{a \in B \mid G_{a}=\emptyset\right\}$ is also contained in a Zariski closed subset of $B$ with codimension $\geq 2$. Therefore, if $\Xi_{3}$ had an irreducible component of codimension $\leq 1$ in $B$, then it must be contained in $f(\operatorname{Sing} f)$ and hence in $\Sigma_{f}$. Because $G_{\zeta(i)}$ is non-empty and disjoint from $\operatorname{Sing} f, \zeta(i)$ is not contained in $\Xi_{3}$. Hence $\Xi_{3}$ is of codimension $\geq 2$ in $B$. Therefore the condition $(\mathrm{S})$ is satisfied.

\section{Proof of Theorem 1.2}

For a line bundle $L \rightarrow C$ of degree $d$ on $C$, we denote by $[L] \in \operatorname{Pic}^{d}(C)$ the corresponding point of the Picard variety. We consider the natural morphism

$$
\bar{\tau}: \operatorname{Sym}^{d} C \rightarrow \operatorname{Pic}^{d}(C),
$$

which is smooth and projective. The fiber $\bar{\tau}^{-1}([L])$ of $\bar{\tau}$ over $[L] \in \operatorname{Pic}^{d}(C)$ is identified with the projective space

$$
P_{L}^{\vee}=\mathbb{P}_{*} H^{0}(C, L)
$$

of one-dimensional linear subspaces of $H^{0}(C, L)$. The dual hypersurface $C_{L}^{\vee} \subset$ $P_{L}^{\vee}$ of the curve $C_{L} \subset P_{L}$ is the intersection of $\bar{\tau}^{-1}([L])$ with the hypersurface $\Delta_{C}^{d} \subset \operatorname{Sym}^{d} C$. We equip $\Delta_{C}^{d}$ with the reduced structure. The degree of $C_{L}^{\vee}$ does not depend on $[L]$. Hence the scheme-theoretic intersection of $\bar{\tau}^{-1}([L])$ and $\Delta_{C}^{d}$ is reduced for any $[L] \in \operatorname{Pic}^{d}(C)$. We denote by $\tau$ the restriction of $\bar{\tau}$ to $\operatorname{Sym}^{d} C \backslash \Delta_{C}^{d}$. Then Theorem 1.1 implies that the sequence

$$
1 \longrightarrow \pi_{1}\left(P_{L}^{\vee} \backslash C_{L}^{\vee}\right) \longrightarrow \pi_{1}\left(\operatorname{Sym}^{d} C \backslash \Delta_{C}^{d}\right) \stackrel{\tau_{*}}{\longrightarrow} \pi_{1}\left(\operatorname{Pic}^{d}(C)\right) \rightarrow 1,
$$

is exact for a general $[L] \in \operatorname{Pic}^{d}(C)$, because $\pi_{2}\left(\operatorname{Pic}^{d}(C)\right)=0$. Therefore $\pi_{1}\left(P_{L}^{\vee} \backslash\right.$ $\left.C_{L}^{\vee}\right)$ is isomorphic to the kernel of the natural homomorphism

$$
B(C, d) \rightarrow H_{1}(C, \mathbb{Z})
$$

because $\tau_{*}$ is identified with this homomorphism.

\section{References}

[1] J. S. Birman, On braid groups, Comm. Pure Appl. Math. 22 (1968), 41-72. 
[2] J. S. Birman, Mapping class groups and their relationship to braid groups, Comm. Pure Appl. Math. 22 (1969), 213-238.

[3] I. Dolgachev and A. Libgober, On the fundamental group of the complement to a discriminant variety, Algebraic geometry (Chicago, Ill., 1980), 1-25, Springer, Berlin, 1981.

[4] W. Fulton and R. Lazarsfeld, Connectivity and its applications in algebraic geometry, Algebraic geometry (Chicago, Ill., 1980), 26-92, Springer, Berlin, 1981.

[5] M. Golubitsky and V. Guillemin, Stable mappings and their singularities, Graduate Texts in Mathematics, Vol. 14, Springer-Verlag, New York, 1973.

[6] J. Kaneko, On the fundamental group of the complement to a maximal cuspidal plane curve, Mem. Fac. Sci. Kyushu Univ. Ser. A 39 (1985), no. 1, 133-146.

[7] V. S. Kulikov and I. Shimada, On the fundamental groups of complements to dual hypersurfaces of projective curves, preprint, MPI 1996 - 32 (1996).

[8] M. V. Nori, Zariski's conjecture and related problems, Ann. Sci. École Norm. Sup. (4) 16 (1983), no. 2, 305-344.

[9] G. P. Scott, Braid groups and the group of homeomorphisms of a surface, Proc. Cambridge Philos. Soc. 68 (1970), 605-617.

[10] I. Shimada, Fundamental groups of open algebraic varieties, Topology 34 (1995), no. 3, 509-531.

Ichiro Shimada

Department of Mathematics

Hokkaido University

Sapporo 060-0810

Japan

e-mail: shimada@math.sci.hokudai.ac.jp

(Received: October 3, 2001)

\section{(D) To access this journal online:}

(LP) http://www.birkhauser.ch 